\title{
Automatic Preference Mining through Learning User Profile with Extracted Information
}

\author{
Kyung-Yong Jung ${ }^{1}$, Kee-Wook Rim² ${ }^{2}$, and Jung-Hyun Lee ${ }^{3}$ \\ ${ }^{1}$ HCI Lab., Dept. of Computer Science \& Information Engineering, Inha Univ., Korea \\ kyjung@gcgc.ac.kr \\ ${ }^{2}$ Dept. of Knowledge Information \& Industrial Engineering, Sunmoon Univ., Korea \\ rim@omega.sunmoon.ac.kr \\ ${ }^{3}$ Dept. of Computer Science \& Information Engineering, Inha Univ., Korea \\ jhlee@inha.ac.kr
}

\begin{abstract}
Previous Bayesian classification has a problem because of reflecting semantic relation accurately in expressing characteristic of web pages. To resolve this problem, this paper proposes automatic preference mining through learning user profile with extracted information. Apriori algorithm extracts characteristic of web pages in form of association words that reflects semantic relation and it mines association words from learning the ontological user profile. Our prototype personalized movie recommender system, WebBot, extracts information about movies from web pages to recommend titles based on training movie set supplied by an individual user. The proposed method was tested in database that users estimated the preference about web pages, and certified that was more efficient than existent methods.
\end{abstract}

\section{Introduction}

Recommender systems using information filtering accumulates a database of users preferences, and then uses them to make personalized recommendations for items such as books, music, clothing, and movies. The user's preference can be either explicit ratings or implicit usage history. Information filtering can help E-commerce in converting web surfers into buyers by personalization of the web interface. It can also improve cross-sell by suggesting other items the user might be interested. In a real world where an E-commerce recommender system's competitors are only a one click, gaining users loyalty is an essential business strategy. Recommender system using information filtering has been very successful in both practice and research. However, there still remain important research issues in overcoming two fundamental challenges for information filtering [5]. The first challenge is to improve the scalability of the information filtering algorithms. Existing information filtering algorithms can deal with thousands of users within a reasonable time, but the demand of modern E-Commerce system is to handle tens of millions of users. The second challenge is to improve the quality of the recommendations. Users need recommendations they can trust to help them find items they will like. If a user trusts a recommender system,

\footnotetext{
A. Fred et al. (Eds.): SSPR\&SPR 2004, LNCS 3138, pp. 815-823, 2004.

(C) Springer-Verlag Berlin Heidelberg 2004
} 
purchases an item, but finds out he does not like the item, the user will be unlikely to use the recommender system again [18]. In this paper, we present automatic preference mining through learning the ontological user profile with extracted information to improve the scalability and the quality of the personalized movie recommender system [7]. Users provide actual 1-6 ratings for a selected training movies set; the system then learns an ontological user profile using Naïve Bayesian algorithm and produces a ranked list of the most recommended additional titles [6,10].

\section{Extracted Information Based on Association Word Knowledge}

We have been exploring the personalized movie recommender system by applying automated text categorization methods to semi-structured text extracted from the web pages [14]. Our current prototype system, WebBot: Web Robot Agent [6,7], uses a database of movie content information extracted from web pages at Internet Movie Database. Therefore, the personalized movie recommender system's content information about titles consists of textual meta-data rather than the actual text of the web pages. An IMDb subject search is performed to obtain a list of movie-description URLs of broadly relevant titles. WebBot then downloads each of these pages and uses a pattern-based information extraction system to extract the data about each title. Information extraction is the task of locating specific information from web pages, thereby getting useful structured related data from unstructured text. Specifically, it involves finding a set of sub-strings from the web pages, for each of a set of slots.

\subsection{Building a Database with Extracting Information}

A WebBot follows the IMDb link provided for every movie in the EachMovie dataset [9] and collects information from the various links off the main URL. We represent the content information of all movies as a set of slots. Each slot is represented as a bag of association words. This content information, after suitable preprocessing such as elimination of stop word etc., is collected into a vector of bag of association words, one bag for each feature describing the movie. IMDb produces the information about related directors and movie titles using information filtering: however, WebBot treats them as additional content information about the movie. Since the layout of IMDb's automatically generated web pages is so regular. A moderately simple extraction system is sufficient. The text in each slot is then processed into an unordered bag of words and all movies represented as a vector of bags of association words [6].

\subsection{Expression of Web Pages Characteristics}

To express the characteristics of web pages as either a bag-of-words or a bag-of association-words [1], it is a necessary preprocess the web pages by analyzing its morphology. The system used in the morphological analysis is identical to the focused 
intelligent information retrieval system [17]. The Apriori algorithm [1] is used to mine related data from the words extracted from morphological analysis. The associated word mining algorithm, Apriori is used to find the associative rules of items out of the set of transactions. The mimed data, or the set of associated words from each web page, are represented as a related-word vector model. As a result, the web page $\left\{d_{j}\right\}$ is represented as Equation (1) in the form of a related-word vector model.

$$
\begin{gathered}
\left\{d_{j}\right\}=\left\{\left(\mathrm{w}_{11} \& \mathrm{w}_{12} \ldots \& \mathrm{w}_{1(\mathrm{r}-1)} \rightarrow \mathrm{w}_{1 \mathrm{r}}\right),\left(\mathrm{w}_{21} \& \mathrm{w}_{22} \ldots \& \mathrm{w}_{2(\mathrm{r}-1)} \rightarrow \mathrm{w}_{2 \mathrm{r}}\right), \ldots,\right. \\
\left.\left(\mathrm{w}_{\mathrm{k} 1} \& \mathrm{w}_{\mathrm{k} 2} \ldots \& \mathrm{w}_{\mathrm{k}(\mathrm{r}-1)} \rightarrow \mathrm{w}_{\mathrm{kr}}\right), \ldots,\left(\mathrm{w}_{\mathrm{p} 1} \& \mathrm{w}_{\mathrm{p} 2} \ldots \& \mathrm{w}_{\mathrm{p}(\mathrm{r}-1)}=>\mathrm{w}_{\mathrm{pr}}\right)\right\}_{j} \quad(j=1,2, \ldots, \mathrm{m})
\end{gathered}
$$

$\left(\mathrm{w}_{\mathrm{k} 1} \& \mathrm{w}_{\mathrm{k} 2} \ldots \& \mathrm{w}_{\mathrm{k}(\mathrm{r}-1)}\right)$ is antecedent of association word $\left(\mathrm{w}_{\mathrm{k} 1} \& \mathrm{w}_{\mathrm{k} 2} \ldots \& \mathrm{w}_{\mathrm{k}(\mathrm{r}-1)} \rightarrow \mathrm{w}_{\mathrm{kr}}\right)$ and $\mathrm{w}_{\mathrm{kr}}$ is consequent of association word $\left(\mathrm{w}_{\mathrm{k} 1} \& \mathrm{w}_{\mathrm{k} 2} \ldots \& \mathrm{w}_{\mathrm{k}(\mathrm{r}-1)} \rightarrow \mathrm{w}_{\mathrm{kr}}\right)$. Here, each of $\left\{\mathrm{w}_{\mathrm{k} 1}, \mathrm{~W}_{\mathrm{k} 2}, \ldots, \mathrm{w}_{\mathrm{k}(\mathrm{r}-1)}, \mathrm{W}_{\mathrm{kr}}\right\}$ in $\left(\mathrm{w}_{\mathrm{k} 1} \& \mathrm{w}_{\mathrm{k} 2} \ldots \& \mathrm{w}_{\mathrm{k}(\mathrm{r}-1)} \rightarrow \mathrm{w}_{\mathrm{kr}}\right)$ represents a word for composing association word. " $p$ " represents the number of association words in a web page. " $r$ " represents the number of words in an association word. The "\&" shows that the words on each side are related. For the good results in extracting related words, the dataset must have a confidence of over 85 and a support of less than 25 [6].

\subsection{Generating the Ontological User Profile}

The association word mining is used to extract features from web pages. Most users have a habit of searching similar or same web pages. We generate an ontological user profile by extracting features from web pages user accesses continuously. Contentbased filtering generates new user profiles by receiving relevance feedback on web pages that users access after receiving recommendations to the web pages. Related words gathered by relevance feedback on web pages the user has visited are stored in the user profile. The related words stored in the user profile, related words show a high frequency is given more weight. The weight means the ratio of each association word to all association words, which are extracted form web pages that user accesses. If $w U P_{a}$ is a user profile with weight given to related words, then $w U P_{a}$ can be expressed as Equation (2).

$$
w U P_{a}=\left\{w_{1} \cdot A W_{1}, w_{2} \cdot A W_{1}, \ldots, w_{t} \cdot A W_{t}\right\}
$$

In Equation (2), $\left\{w_{l}, w_{2}, \ldots, w_{t}\right\}$ is a weight vector that shows the weight of the related word, and $t$ is the total number of related words within the ontological user profile. The ontological user profile is generated based on the web page features extracted from user's preference rating. The preference of association words expressed in features is indicated various values according to the weight.

\section{Automatic Preferences Mining through Learning User Profile}

Previous studies of automatic learning of profile include use of probability [4,8], use of statistics [13] and use of vector similarity [7,8]. Among them, learning user profile through Bayesian probability is effective method [10,15]. Since learning the ontological user profile through use of simple Naïve Bayesian classifier extracts all word 
appeared in web pages, it is hard to reflect characteristic of web pages accurately. Mistaken learning user profile caused by this reduces accuracy of classification. Because of this, Bayesian classification method [11] that uses TF.IDF to make it more accurate was suggested. The suggested method extracts characteristic of web pages through use of TF.IDF from web pages [10]. It also gives weight to characteristic extracted from web pages and so mistaken classification caused by noises is reduced more than simple Naïve Bayesian classifier. However, since characteristic of extracted web pages does not reflect semantic relation, it could not resolve the problem of mistaken classification caused by ambiguity of words [6]. To resolve this problem, this paper suggests automatic preference mining through learning user profile with extracted information. In suggested method, Apriori algorithm [1] extracts characteristic of web pages in the form of association words that reflects semantic relation between words.

\subsection{Learning a User Profile Using Naïve Bayesian Classifier}

The learner currently employed by WebBot [6,7] is a bag of association words Naïve Bayesian classifier [10] extended to handle a vector of bags rather than a single bag. WebBot does not attempt to predict the extract numerical rating of a movie title, but rather just a total ordering or ranking of titles in order of preference $[14,15,16]$.

We use a multinomial text model, in which a web page is modeled as an ordered sequence of ordered sequence of word events drawn from the same vocabulary, $V$. The Naïve Bayesian assumption states that the probability of each word is dependent on the web page classes but independent of the word's context and position. For each class $c_{j}$, and word, $w_{\mathrm{k}} \square V$, the probability, $P\left(c_{j}\right)$ and $P\left(w_{\mathrm{k}} \mid c_{j}\right)$ must be estimated from the training data. Then the posterior probability of each class given a web page, $D$, is computed using Bayes rule by Equation (3).

$$
p\left(c_{j} \mid D\right)=\frac{P\left(c_{j}\right)}{P(D)} \prod_{i=1}^{|D|} P\left(a_{i} \mid c_{j}\right)
$$

Where $a_{\mathrm{i}}$ is the $i$ th word in the web page, and $|D|$ is the length of the page in words. Since for any given page, the prior $P(D)$ is a constant, this factor can be ignored if all that is desired is a rating rather than a probability estimate. A ranking is produced by sorting pages by their odds ratio, $P\left(c_{1} \mid D\right) / P\left(c_{0} \mid D\right)$, where $c_{1}$ represents the positive class and $c_{0}$ represents the negative class. A movie is classified as positive if the odds are greater than 1, and negative otherwise. In case, since movies are represented as a vector of "web pages", $d_{\mathrm{m}}$, one for each slot $s_{\mathrm{m}}$, the probability of each word given the category and the slot $P\left(w_{\mathrm{k}} \mid c_{j}, s_{\mathrm{m}}\right)$, must be estimated and the posterior category probability for a film, $F$, computed using Equation (4).

$$
p\left(c_{j} \mid F\right)=\frac{P\left(c_{j}\right)}{P(F)} \prod_{m=1}^{|S|} \prod_{i=1}^{\left|d_{m}\right|} W_{n k} \cdot P\left(a_{m, i} \mid c_{j}, s_{m}\right)
$$

Where $S$ is the number of slots and $a_{\mathrm{m}, \mathrm{i}}$ is the $i$ th word in the $m$ th slot. The class with the highest probability determines the predicted rating. The Laplace smoothing [10] 
is used to avoid zero probability estimates for words that do not appear in the limited training movie set. Finally, calculation with logarithms of probabilities is used to avoid underflow.

Naïve Bayesian classifier through use of TF.IDF [13] makes morphological analysis of document to extract characteristic of web pages and extracts only nouns from its outcome. TF.IDF of all extracted nouns can be obtained through Equation (5).

$$
W_{n k}=f_{n k} \cdot\left[\log _{2} \frac{n}{D F}+1\right]
$$

In Equation (5), $f_{\mathrm{nk}}$ is relative frequency of word $n_{k}$ against all words within all web pages and $n$ is the number of web pages and DF is the number of learning web pages where word $n_{k}$ appeared. If characteristic of web pages is $\left\{a_{1} a_{2, \ldots} a_{i, \ldots .} a_{D}\right\}$, Naïve Bayesian classifier classifies web pages into one class among $\left\{c_{1}, c_{2, \ldots, \ldots, \ldots,} c_{i, \ldots} c_{j}\right\}$. Equation (6) is used to give probability of association word $\left(w_{\mathrm{k} 1} \& w_{\mathrm{k} 2} \ldots \& w_{\mathrm{k}(\mathrm{r}-1)} \rightarrow w_{\mathrm{kr}}\right)$ within $c_{\mathrm{j}}, s_{\mathrm{m}}$ is expressed as $\left.p\left(\left(w_{\mathrm{k} 1} \& w_{\mathrm{k} 2} \ldots \& w_{\mathrm{k}(\mathrm{r}-1)}\right) \rightarrow w_{\mathrm{kr}}\right) \mid c_{\mathrm{j}}, s_{\mathrm{m}}\right)$. If an association word appears $n$ times in a movie $F_{e}$, it is counted as occurring $a_{e 1} n$ times in a positive movie and $a_{e 0} n$ times in a negative movie. The parameters are estimated as Equation (6).

$$
p\left(\left(\mathrm{w}_{\mathrm{k} 1} \& \mathrm{w}_{\mathrm{k} 2} \ldots \& \mathrm{w}_{\mathrm{k}(\mathrm{r}-1)} \rightarrow w_{k(r-1)}\right) \mid c_{j}, s_{m}\right)=\sum_{e=1}^{N} W_{n k} \cdot a_{e j} \cdot n_{k e m} /\left(\sum_{e=1}^{N} a_{e j}\left|d_{m}\right|\right)
$$

Where $n_{\text {kem }}$ is the count of the number of times word $w_{k}$, appears in movie $F_{e}$, in slot $s_{\mathrm{m}}$, and denotes the total weighted length of web pages in category $c_{j}$ and slot $s_{\mathrm{m}}$.

\subsection{Automatic Preferences Mining for Recommendations}

To produce recommendations, WebBot [6,7] learns the ontological user profile, predicts the ratings for the un-rated movies, and finally ranks the movies by their ratings. Current WebBot uses one of the three methods to learn an ontological user profile. The first method is a binary Naïve Bayesian classifier. It treats movies rated 1-3 as negative instances, and those rated 4-6 as positive instances. The scores are ranked based on the natural log of the posterior odds of positive. A second method treats the 6 ratings as 6 distinct categories $[8,14,15]$. When predicting for a target movie, the personalized movie recommender system computes the posterior probability of each category given the target movie. Then the predicted rating of the posterior probability distribution for the categories is computed and used as the predicted ratings by Equation (7). Here, $P(i)$ is the posterior probability for category $i$.

$$
\text { predicted score }=\sum_{i=1}^{6} i \cdot P(i)
$$

We use the predicted ratings rather than choosing the most probable category to better represent the continuity of the ratings. When using the 6-category classifier to predict a binary category, we classify all movies as positive category or negative category. The final method is used a weighted binary classifier that maps the user's actual 1-6 rating into a weight value. As with relevance feedback in information retrieval, this cycle can be repeated several times to recommend the good results $[2,10,13,15]$. 


\section{Performance Evaluations}

We use the user-movie ratings data provided by the EachMovie dataset and the movie details from the Internet Movie Database (www.imdb.com). We represent the content information of every movie as a set of slots. Each slot is represented as a bag of association words. The slots we use for the EachMovie dataset [9] are: movie title, cast, director, newsgroup reviews, genre, plot, summary, plot keywords, user comments, external reviews, and award. The reduced dataset has 299,997 ratings for 1,289 movies for which content information was available from IMDb. To evaluate various approaches of information filtering, we divided the rating dataset in test-set and training-set. To observe performance given varying amounts of training movie set, learning curves were generated by checking the recommender system after training on increasing subsets of the overall training data. A number of metrics were used to performance measures, mean absolute error (MAE) and rank scoring measure (RSM), both suggested by [3], F-measure are used to gauge performance. It is important to evaluate accuracy and recall in conjunction. To quantify this with single measure, we use F-measure in Equation (8), which is a weighted combination of accuracy and recall $[6,8,10]$. $P$ indicates accuracy and $R$ means recall and in that case, the higher Fmeasure the better classification. Beta indicates relative weight of recall against accuracy and if it is 1.0, weight of accuracy and recall is the same. " $a$ " is the number of web pages, which appear in both classes. " $b$ " is the number of web pages, which appear in class categorized by first method but not in class categorized by second method. " $c$ " is the number of web pages, which appear in class categorized by second method but not in class categorized by first method. In this experiment, beta was designed as 1 to analysis the results of classification, and different results of Fmeasure according to change in value of beta from 0.5 to 1.4 were observed [6,7].

$$
F-\text { measure }=\frac{\left(\beta^{2}+1\right) P R}{\beta^{2} P+R} \quad P=\frac{a}{a+b} 100 \% \quad R=\frac{a}{a+c} 100 \%
$$

Fig. 1 shows the result of MAE, RSM at varying the training movie set. For current experiments, this paper compares the following methods: a simple binary classifier, a 6-ratings classifier, and a weighed binary classifier.

We expected that the binary classifier would perform better on classification accuracy on the MAE, RSM since it is specifically designed for that task. Since users will often be willing to rate a small number of the training movie set, getting enough ratings to produce good performance from the 6-ratings classifier could often be impractical. However, as the weighted binary classifier performs comparable to the binary classifier, this proposes that the weighted binary classifier may be the good [15].

For evaluation, this paper uses all of the following methods: the proposed method using learning user profile with extracted information (LUP_EI), the collaborative filtering method using the Pearson correlation coefficient (P_Corr) [3], the recommendation method using only the content-based filtering (Content) [4], and naïve combined approach (N_Com) [16]. The naïve combined approach takes the average of the ratings generated by collaborative filtering and content-based filtering. These methods are compared by varying the number of clustered users. Also, the proposed 

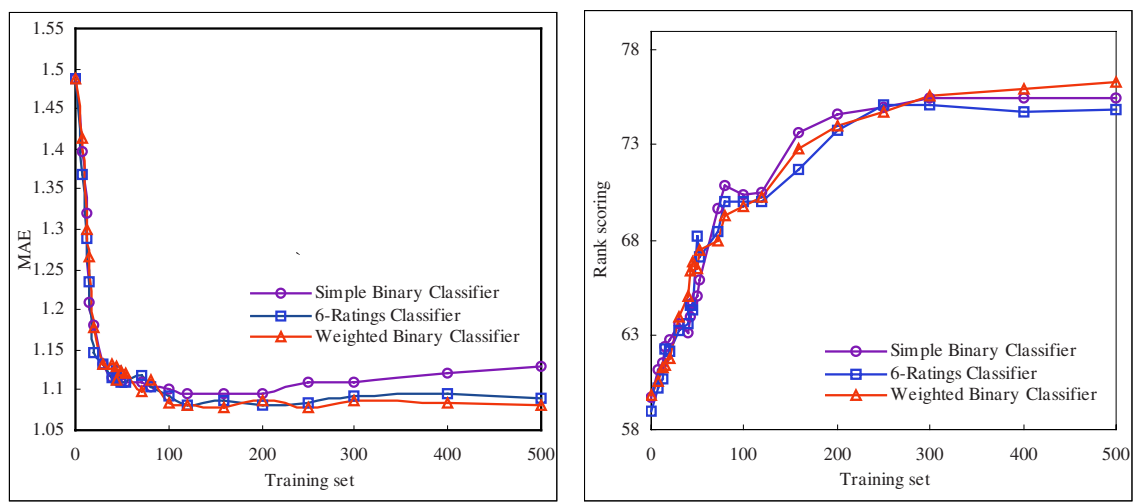

Fig. 1. MAE, RSM at varying the training movie set.
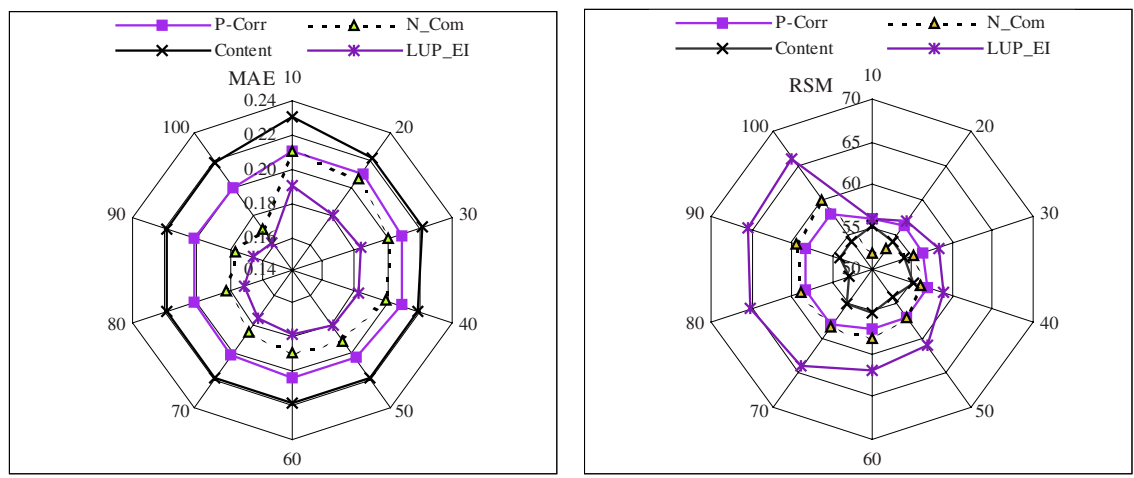

Fig. 2. MAE, RSM at varying the number of clustered users.

method (LUP_EI) was compared with the previous methods that use both collaborative filtering and content-based filtering by changing the number of user evaluations on items. The previous methods include the Pazzani method [12], Lee method [8], and Good method [5] using user profile.

Fig. 2 shows the MAE and RSM of LUP_EI, P_Corr, Content, and N_Com. Fig. 2, as the number of users increases, the performance of the LUP_IE, and the N_Com also increases, whereas P_Corr and Content show no notable change in performance. In terms of accuracy of prediction, it is evident that method LUP_EI is more superior to others. Fig. 3 shows the prediction speed of LUP_EI, Pazzani method, Lee method, and Good method when the number of user's evaluations is increased. Fig. 4 shows the result of F-measure at varying with change for with Beta. Fig. 3 indicates speed of prediction at $n$th rating about 1,892 movies. LUP_EI is the fastest $8.71 \mathrm{sec}$ followed by $10.84 \mathrm{sec}$ of Pazzani method, 14.7 sec of Lee method, and 13.2sec of Good method. In terms of speed, LUP_EI and Pazzani are excellent in comparison with others and remainder records similar speed. Fig. 4 indicates analyzed performance of F-measure according to change of beta value from 0.5 to 1.4. LUP_EI represents a rising curve as beta value increases and so it records better performance in terms of accuracy than 


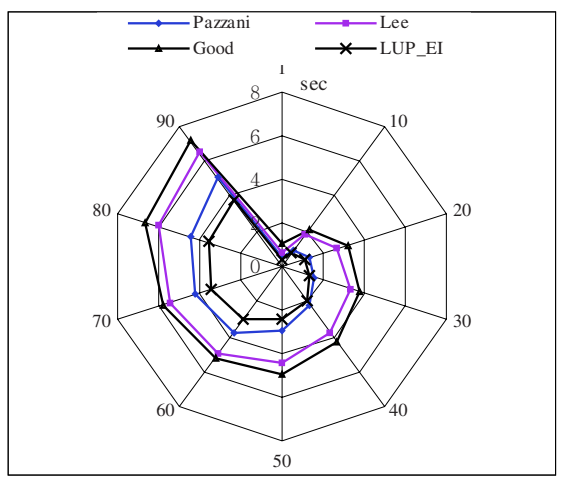

Fig. 3. Prediction speed at $n$th rating (sec).

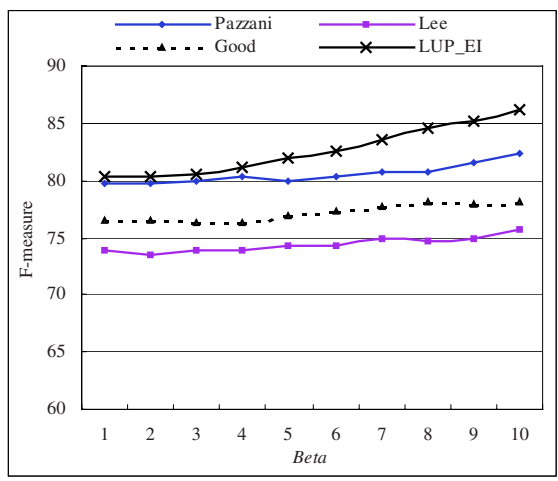

Fig. 4. F-measure at varying Beta.

recall. But in Pazzani method and Good method, change of beta value hardly affects F-measure value and so it has similar level of performance in terms of recall and accuracy. However, in Lee method, it has a bit higher performance in terms of recall than accuracy. On average, if beta is 1.0, LUP_EI has $1.31 \%$ higher performance than Pazzani method and $2.13 \%$ Good method and $4.79 \%$ Lee method.

\section{Conclusions}

Recommender systems improve access to relevant products and information by making personalized suggestions based on previous movies of a user's likes and dislikes. We have proposed automatic preference mining through learning user profile with Naïve Bayesian algorithm to efficiently handle set-valued features. And then information about movies extracted from web pages to recommend movie titles based on training movies supplied by an individual user. The suggested method has two advantages. First, it has refined association words so that Naïve Bayesian classifier effects accurate and speedy classification of web pages. Second, it removes confusion in meaning by expressing characteristic of web pages. As a result, the automatic preference mining through learning user profile with extracted information has $1.31 \%$ higher performance than Pazzani method and 2.13\% higher than Good method and $4.79 \%$ than Lee method. The proposed method was compared with the existent methods rating preference automatically in recommender systems, and the existent methods for information filtering, which the naïve combined method. The proposed method shows higher performance than existent method in both comparisons. Our approach could provide a useful service to customers overwhelmed by the abundance of choice presented by the world.

\section{Acknowledgements}

The author would like to thank the anonymous reviewers whose comments helped improve the paper. This work was supported by INHA UNIVERSITY Research Grant. (INHA-31613) 


\section{References}

1. R. Agrawal and R. Srikant, "Fast Algorithms for Mining Association Rules," In Proc. of the 20th International Conference Very Large Data Bases, pp.487-499, Santiago, Chile, 1994.

2. D. Billsus, M. J. Pazzani, "Learning Collaborative Information Filters," In Proc. of the 15th International Conference on Machine Learning, pp.46-54, 1998.

3. J. S. Breese, D. Heckerman, C. Kadie, "Empirical Analysis of Predictive Algorithms for Collaborative Filtering," In Proc. of the Conference on Uncertainty in Artificial Intelligence, pp.43-52, Madison, WI, 1998.

4. D. D. Lewis, "Naive (Bayes) at forty: The Independence Assumption in Information Retrieval," Proc. of the 10th European Conference on Machine Learning, pp.4-15, 1998.

5. N. Good, et al., "Combining Collaborative Filtering with Personal Agents for Better Recommendations," In Proc. of the 16th National Conference on Artificial Intelligence, pp.439-446, 1999.

6. S. J. Ko, J. H. Lee, "Bayesian Web Document Classification through Optimizing Association Word," In Proc. of the 16th International Conference on Industrial and Engineering Applications of Artificial Intelligence and Expert Systems, LNAI 2718, Springer-Verlag, pp.565-574, 2003.

7. K. Y. Jung, J. H. Lee, "Hybrid Collaborative Filtering and Content-based Filtering for Improved Recommender System," In Proc. of the International Conference on Computational Science, LNCS, Springer-Verlag, pp.299-306, 2004.

8. W. S. Lee, "Collaborative Learning for Recommender Systems," In Proc. of the 18th International Conference on Machine Learning, pp.314-321, 1997.

9. P. McJones, EachMovie dataset, URL: http://www.research.digital.com/SRC/eachmovie

10. T. Mitchell, Machine Learning, McGraw-hill, New York, pp.154-200, 1997.

11. Introduction to Rainbow URL: http://www.cs.cmu.edu/afs/cs/project/theo-11/www/naïvebayes.html.

12. M. J. Pazzani, "A Framework for Collaborative, Content-based and Demographic Filtering," Artificial Intelligence Review, pp.393-408, 1999.

13. T. Joachims, "A Probabilistic Analysis of the Rocchio Algorithm with TF.IDF for Text Categorization," In Proc. of the 14th International Conference on Machine Learning, pp.143-151, 1997.

14. P. Melville, et al., "Content-Boosted Collaborative Filtering for Improved Recommendations," In Proc. of the National Conference on Artificial Intelligence, pp.187-192, 2002.

15. R. Raymond, P. Bennett, L. Roy, "Book Recommending Using Text Categorization with Extracted Information," In Proc. of the AAAI'98/ICML Workshop on Learning Categorization, pp.49-54, Madison, WI, 1998.

16. I. Soboroff, C. Nicholas, "Combining Content and Collaboration in Text Filtering," In Proc. of the IJCAI'99 Workshop on Machine Learning in Information Filtering, pp.86-91, 1999.

17. P. N. Bennett, et al., "Probabilistic Combination of Text Classifiers using Reliability Indicators: Models and Results," In Proc. of the 25th Annual International ACM SIGIR Conference, pp.207-214, 2002.

18. K. Yu, et al., "Feature Weighting and Instance Selection for Collaborative Filtering: An Information-Theoretic Approach," Knowledge and Information Systems, Vol. 5, No. 2, pp. 201-224, Springer-Verlag, 2003. 